\title{
Design Rules for Manganites with Novel Magnetic and Electronic Properties
}

\author{
B. Dabrowski*, O. Chmaissem, J. Mais and S. Kolesnik \\ Department of Physics, Northern Illinois University, DeKalb, IL 60115, USA
}

We have been systematically developing strategies for making new perovskite manganites with novel magnetic and electronic properties. This effort requires a two-fold approach: understanding the dependence of properties on chemical and structural factors and development of the ability to synthesize desired compounds. We show that, similar to other single valent $3 d$ systems, the magnetic superexchange interactions in $\mathrm{AMnO}_{3}$ manganites $(\mathrm{A}=$ rare or alkaline earth's) are dependent on the $\mathrm{Mn}-\mathrm{O}-\mathrm{Mn}$ bond angle which is a function of interatomic distances $\mathrm{A}-\mathrm{O}$ and $\mathrm{Mn}-\mathrm{O}$. The local structural disorder on the A-site suppresses magnetic interactions. Recently, by comparing disordered (randomly mixed $\mathrm{La} / \mathrm{Ba}$ ) and ordered (forming $\mathrm{Ba} / \mathrm{La} / \mathrm{Ba}$ layers along the $c$-axes) perovskites we have demonstrated much more conspicuous effects of structural and charge disorder for mixed-valent manganites. We show that by stabilizing the ordered structure; i.e., by suppressing local structural and charge disorder on the A-site, a substantial increase in $T_{\mathrm{C}}$ from 340 to $365 \mathrm{~K}$ can be achieved. A similar control of order on the Mn-site was achieved for $\mathrm{SrMn}_{1-y} \mathrm{Ga}_{y} \mathrm{O}_{3-y / 2}$ compounds near $y=0.5$, while $\mathrm{SrMn}_{1-y} \mathrm{Fe}_{y} \mathrm{O}_{3}$ compounds are always randomly mixed. To achieve these compounds, we have been systematically developing special synthesis techniques for extending chemical composition ranges far beyond those previously achieved, developing rules for predicting which compositions should be possible to produce by these special techniques, and establishing methods for selectively ordering or disordering mixtures of metal atoms on the A- or Mn-sites.

PACS numbers: $61.12 .-\mathrm{q}, 75.50 .-\mathrm{y}$

\section{Introduction}

Perovskite and perovskite related compounds, $\mathrm{ABO}_{3}$, have been recently studied in depth because of intriguing magnetic and electronic properties result-

*corresponding author; e-mail: dabrowski@anl.gov 
ing from competing charge, exchange, and phonon interactions [1]. The properties can be tuned over a wide range through the choice of the sizes and charges of the large size 12-coordinated A cations. These ions control the degree of structural distortions and the formal valence of the smaller size 6-coordinated B ions. For the vast majority of perovskite compounds the B-site ions are moderately electronegative forming mixed covalent/ionic bonds with oxygen while the A-site ions are strongly electropositive forming ionic bonds. Consequently, the valence electrons are transferred from the A ions to the 3-dimensional network of B- and oxygen-ions. The $\mathrm{B}-\mathrm{O}_{3}$ network is built from relatively rigid $\mathrm{BO}_{6}$ octahedra sharing corners and forming nearly straight $\mathrm{B}-\mathrm{O}-\mathrm{B}$ bond-angles. Here, the $\mathrm{B}$-site ions of interest are the Mn and other transition metals that exhibit partially filled $3 d$ electronic orbitals. Thus, the electronic and magnetic properties of perovskite compounds are almost completely determined by the properties of the $\mathrm{B}-\mathrm{O}_{3}$ network whereas the charge state of the network and its structural distortions are fixed by the kind of ions distributed over A-sites. We have been systematically developing an understanding of the relationship between properties of perovskite compounds and their chemical composition, structure, and synthesis conditions to enable the preparation of improved materials and to discover new, exciting compounds. In this paper we review strategies for designing, making, and measuring new perovskite manganites with enhanced magneto-resistive properties and increased magnetic transition temperatures. This effort requires a two-fold approach: understanding the dependence of properties on chemical and structural factors and development of ability for synthesis of desired compounds.

To develop a perceptible description of magnetism and spin-transport in manganites, we will use concepts of local sites of individual magnetic ions rather than the band structure theory. This approach is justified by the inability of current band structure calculations to capture all aspects of the strong coupling among electrons, spins, and phonons as well as to describe strong correlations of the $3 d$ electrons in distorted cubic crystal fields [2]. Thus, we are interested in a conceptually simple description based on the ionic model that grasps most of essential physics rather than the complex calculations that require ample computing power and extensive computing and analysis time. Our description will use internal structural parameters of the $\mathrm{Mn}-\mathrm{O}-\mathrm{Mn}$ bond angles and the $\mathrm{A}-\mathrm{O}$ and $\mathrm{Mn}-\mathrm{O}$ bond lengths that are obtained from neutron diffraction measurements. These structural measurements are combined with detailed magnetic and resistive measurements that are performed over a wide temperature range to provide accurate composition-structure-property phase diagrams that can be used to discover better materials for research and application.

The correlations between structural parameters and physical properties are frequently described in terms of the average structure using a concept of tolerance factor $t=[\mathrm{A}-\mathrm{O}] /(\sqrt{2}[\mathrm{Mn}-\mathrm{O}])[3]$. Here $[\mathrm{A}-\mathrm{O}]$ and $[\mathrm{Mn}-\mathrm{O}]$ are the average cation-oxygen interatomic distances of the A- and B-sites, respectively, the ap- 
proximate values of which can be obtained from the ionic sizes that are tabulated for various coordination numbers and oxidation states at room temperature [4]. We have recently shown that the tolerance factor can be also used to describe the structural stability of the perovskite phase when the effects of varying synthesis temperature and oxygen content are correctly included [5]. Knowledge of the dependence of tolerance factor on composition, temperature, and oxygen content allows us to predict which compositions should form for the A- and B-site substituted manganites. To achieve these compounds, we have been systematically developing special synthesis techniques for extending chemical composition ranges far beyond those previously achieved and establishing methods for selectively ordering or disordering mixtures of metal atoms on the A- or B-sites. Our design rules for the synthesis of perovskite compounds enable the chemical compositions and crystal structures of these materials to be controlled in order to achieve the desired magnetic and electronic properties.

\section{Basics of the electronic configurations and exchange interaction for perovskite manganites}

The range of the Mn oxidation states displaying remarkable magneto-resistive properties for the perovskite compounds $\mathrm{ABO}_{3-\delta}$ spans from $\mathrm{Mn}^{2+}$ through $\mathrm{Mn}^{3+}$ to $\mathrm{Mn}^{4+}$. In the cubic crystal field, which is characteristic of perovskites, these oxidation states of Mn display well-defined ionic ground states of $t^{3} e^{2}, t^{3} e^{1}$, and $t^{3} e^{0}$ for $\mathrm{Mn}^{2+}, \mathrm{Mn}^{3+}$, and $\mathrm{Mn}^{4+}$, respectively. These single-valent states can be achieved by using rare earth's $(\mathrm{R}=\mathrm{La}, \mathrm{Pr}, \mathrm{Nd}, \ldots)$ and alkaline earth's (Ca, Sr, and $\mathrm{Ba}$ ) on the $\mathrm{A}$-sites as well as by introducing oxygen vacancies, $\delta$. The filling of atomic electronic $d$-orbitals, $t_{2 \mathrm{~g}}$ and $e_{\mathrm{g}}$, with electrons that is consistent with Hund's and crystal-field splitting rules has been described in several textbooks [6]. For future reference we only need to recall here that the ionic size of Mn rapidly decreases with its increasing oxidation state because of the decreased filling of the $e_{\mathrm{g}}$ orbitals. And, in addition, that the $\mathrm{Mn}^{4+}$ ion with three electrons in three different orbitals $t_{2 \mathrm{~g}}$ is nearly spherically symmetric while the $\mathrm{Mn}^{3+}$ ion with a single electron in two different orbitals $e_{\mathrm{g}}$ is highly asymmetric because of the Jahn-Teller distortion associated with removal of the two-fold degeneracy of the $e_{\mathrm{g}}$ orbitals.

Now, because in the perovskite structure each Mn pair is separated by an oxygen ion, there are no significant direct magnetic interactions between local $J=$ $5 / 2,4 / 2$, and $3 / 2$ moments of $\mathrm{Mn}^{2+}, \mathrm{Mn}^{3+}$, and $\mathrm{Mn}^{4+}$, respectively. In addition, because the spin-orbit coupling is quenched in the perovskite structure [2], the antiferromagnetic (AF) and ferromagnetic (FM) indirect superexchange describes very well the coupling between magnetic moments for the single-valent states. The Goodenough-Kanamori rules of superexchange interactions are used to predict the sign of the interaction for straight $\mathrm{Mn}-\mathrm{O}-\mathrm{Mn}$ bond angles [7]. When the $\mathrm{Mn}-\mathrm{O}-\mathrm{Mn}$ 
bonds are not straight, the interactions can be described as a superposition of 180 and $90 \mathrm{deg}$ bonds [8]. The correlation of antiferromagnetic transition temperatures to the $\mathrm{Mn}-\mathrm{O}-\mathrm{Mn}$ bond-angle $\theta, T_{\mathrm{N}} \approx T_{\mathrm{Nmax}}-T_{1}\left(1-\left\langle\cos ^{2} \theta\right\rangle\right)$, has been clearly established for single valent $\mathrm{RBO}_{3}$ systems with $\mathrm{B}=\mathrm{Fe}^{3+}\left(t^{3} e^{2}\right)$ and $\mathrm{Cr}^{3+}\left(t^{3}\right)$ when there is only one kind of rare earth on the A-site $[8,9]$.

\section{Magnetic transitions and structural features for single-valent systems}

We have recently shown that a similar relationship between the $\mathrm{Mn}-\mathrm{O}-\mathrm{Mn}$ bond-angle and $T_{\mathrm{N}}$ holds for the $\mathrm{RMnO}_{3}$ perovskites with a single valent $\mathrm{Mn}^{3+}$ $\left(t^{3} e^{1}\right)$ while for the solid solution system $\mathrm{Sr}_{1-x} \mathrm{Ca}_{x} \mathrm{MnO}_{3}$ with $\mathrm{Mn}^{4+}\left(t^{3}\right)$, the structural disorder on the $\mathrm{A}$-site introduces a measurable deviation from this relationship [5, 10]. Figure 1a shows a schematic phase diagram of the magnetic, resistive, and structural properties versus $\left\langle\cos ^{2} \theta\right\rangle$ for $\mathrm{RMnO}_{3}$ perovskites. The complex AF A-type magnetic structure of these compounds is a result of the Jahn-Teller distortion and the orbital order present below temperatures, $T_{\mathbf{J T}}$ [11]. The phase diagram of $\mathrm{RMnO}_{3}$ perovskites is notably similar to the phase diagram of $\mathrm{RNiO}_{3}$ (see Fig. 1b) [12]. Both these phase diagrams show that the $T_{\mathrm{N}} \approx\left\langle\cos ^{2} \theta\right\rangle$ dependence is observed even for complex interactions of the Jahn-Teller B-site ions like $\mathrm{Mn}^{3+}$ with orbital order and mixed $\mathrm{AF}$ and $\mathrm{FM}$ interactions and for $\mathrm{Ni}^{3+}\left(t^{6} e^{1}\right)$ with charge separation, orbital order, and mixed AF and FM interactions.
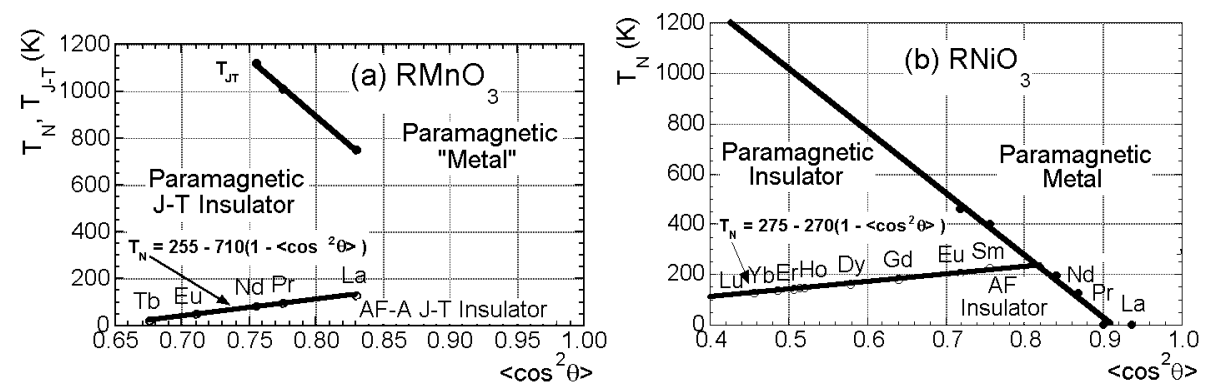

Fig. 1. Phase diagram of the magnetic $T_{\mathrm{N}}$, resistive $T_{\mathrm{JT}}$, and structural properties versus $\left\langle\cos ^{2} \theta\right\rangle$ for $\mathrm{RMnO}_{3}$ (a) and $\mathrm{RNiO}_{3}$ (b) perovskites.

The linear dependence of $T_{\mathrm{N}}$ on $\left\langle\cos ^{2} \theta\right\rangle$ observed for $\mathrm{RMnO}_{3}$ is less regular for the solid solution system $\mathrm{Sr}_{1-x} \mathrm{Ca}_{x} \mathrm{MnO}_{3}$. The size difference between interatomic distances of the $\mathrm{Sr}-\mathrm{O}$ and $\mathrm{Ca}-\mathrm{O}$ (for the isovalent $\mathrm{Sr}$ and $\mathrm{Ca}$ cations) introduces local variance of the bond angles that suppresses $T_{\mathrm{N}}$ by $\sim 20 \mathrm{~K}$ at $x=0.5$, where the variance of sizes is the largest [13]. The properties phase diagrams have been most frequently described in terms of the average structure using the tolerance factor, $t(x)$. It was reported recently that the ferromagnetic transition temperatures of the mixed-valent manganites can be better modeled 
by using the variance of sizes of the A-site ions in addition to the tolerance factor or interatomic distances, $s(x)=\sqrt{\Sigma_{i}\left\{x_{i}\left[\mathrm{~A}_{i}-\mathrm{O}\right]^{2}-[\mathrm{A}-\mathrm{O}]^{2}\right\}}$, where $[\mathrm{A}-\mathrm{O}]=$ $\Sigma_{i} x_{i}\left[\mathrm{~A}_{\mathrm{i}}-\mathrm{O}\right]$, and $x_{i}$ is the fractional occupancy of atoms occupying the A-site [14]. We have used our own measured interatomic distances $[\mathrm{Ca}-\mathrm{O}]=2.640$ and $[\mathrm{Sr}-\mathrm{O}]$ $=2.691 \AA$ to approximate the variation of the local $\mathrm{Mn}-\mathrm{O}-\mathrm{Mn}$ bond angles from its average by the variance of sizes of the A-site ions, $s(x=0.5)=0.026 \AA[5,15]$. The observed suppression of $T_{\mathrm{N}}$ and $T_{\mathrm{C}}$ by the variance of sizes shows that the magnetic transition temperatures are very sensitive to the local variation of the $\mathrm{A}-\mathrm{O}$ bond lengths and $\mathrm{Mn}-\mathrm{O}-\mathrm{Mn}$ bond angles.

\section{Synthesis of kinetically stable perovskites}

The tolerance factor $t(x)=[\mathrm{A}-\mathrm{O}] /(\sqrt{2}[\mathrm{Mn}-\mathrm{O}])$, rather than the $\mathrm{Mn}-\mathrm{O}-\mathrm{Mn}$ bond angles, is usually used to describe physical properties of compounds $\mathrm{A}_{1-x} \mathrm{~A}_{x}^{\prime} \mathrm{MnO}_{3}$ with varying compositions $x$ at room temperature [16]. To improve the calculation of the tolerance factor we have used neutron powder diffraction to precisely measure individual $\mathrm{A}-\mathrm{O}$ and $\mathrm{Mn}-\mathrm{O}$ bond lengths at $300 \mathrm{~K}$ for $\mathrm{Sr}_{1-x} \mathrm{Ca}_{x} \mathrm{Mn}^{4+} \mathrm{O}_{3}$ and $\mathrm{La}_{1-x} \mathrm{Sr}_{x} \mathrm{Mn}^{3+x} \mathrm{O}_{3}[5,17]$. Individual bond lengths were used to derive average equilibrium interatomic distances specific for perovskite manganites $[\mathrm{A}-\mathrm{O}],\left[\mathrm{Mn}^{3+-} \mathrm{O}\right]=1.98$ and $\left[\mathrm{Mn}^{4+} \mathrm{O}\right]=1.896 \AA$ instead of relying on tabulated values of ionic radii obtained for different compounds [4]. Knowledge of accurate interatomic distances is of great value for crystallographers and physicists since the average sizes of $[\mathrm{A}-\mathrm{O}]$ and $[\mathrm{B}-\mathrm{O}]$ can be reliably calculated for any combination of ions to predict $t(x), s(x)$, and describe magnetic and resistive behavior [5].

The tolerance factor is also frequently used to estimate the solubility limits and predict stability of novel compounds. However, because the synthesis is done at high temperatures, $800-1400^{\circ} \mathrm{C}$, it is important to know dependence of the tolerance factor on temperature $T$ and oxygen content $\delta$. This dependence of $t(x, T, \delta)$ on $T$ and $\delta$ arises because the interatomic bond lengths expand with increasing temperature. In addition, the oxygen content is not always constant at these temperatures, such that the formal valence of $\mathrm{Mn}$ is changing, and so is its bond length to oxygen. By using neutron diffraction and thermogravimetric measurements (TGA) over a wide range of temperatures we have estimated the temperature dependence of the tolerance factor for both stoichiometric and oxygen deficient compositions. We have observed that the tolerance factor shows a simple parabolic dependence on $T$ for stoichiometric compounds over a wide range of temperatures $0-600 \mathrm{~K}, t(x, T, \delta=0)=t_{0}(x)+t_{2}(x) T^{2}$. In addition, we have observed that over this temperature range $t(x, T, \delta=0)$ can be used to reliably predict structural phase transitions from cubic to tetragonal at $t=1$ and from tetragonal to orthorhombic at $t=0.996$ as a function of composition and temperature [5]. 
The effect of oxygen nonstoichiometry was included by defining the average Mn-oxygen interatomic distance $\left[\mathrm{Mn}^{4-2 \delta}-\mathrm{O}\right]=(1-2 \delta)\left[\mathrm{Mn}^{4+}-\mathrm{O}\right]+(2 \delta)\left[\mathrm{Mn}^{3+}-\mathrm{O}\right]$. TGA measurements have shown that during heating above $\sim 400^{\circ} \mathrm{C}(\sim 675 \mathrm{~K})$, the oxygen content of the perovskite material $\mathrm{Sr}_{1-x} \mathrm{Ca}_{x} \mathrm{MnO}_{3}$ with compositions $0 \leq x \leq 0.5$ begins to decrease. Rapid variation of oxygen content at $800-1400^{\circ} \mathrm{C}$ was observed, and shown by X-ray diffraction to result from a reversible transformation to a hexagonal 4-layered phase that exhibits a different oxygen environment around Mn. The perovskite phase was observed to be stable at high temperatures only when the oxygen content was in the specific range of $2.50-(3-\delta)$. The oxygen vacancy content $\delta$ necessary to make stable the perovskite phase was found to decrease with the substitution amount, $x$, such that compositions with $x>0.5$ did not transform to the hexagonal phase in air at any temperature. The structural changes between the perovskite and non-perovskite hexagonal phases have been explained using tolerance factor that is a function of composition, temperature, and oxygen content. The dependence of the observed phases on the magnitude of the tolerance factor confirmed that the stability of the perovskite phase is limited by the condition $t(x, T, \delta) \leq 1.0$ at synthesis temperatures $T>\sim 800^{\circ} \mathrm{C}[5]$.

Figure 2 shows the calculated tolerance factor at $1325^{\circ} \mathrm{C}$ as a function of the $[\mathrm{A}-\mathrm{O}]$ bond length (or the composition of $\mathrm{Sr}_{1-x-y} \mathrm{Ca}_{x} \mathrm{Ba}_{y} \mathrm{MnO}_{3}$ ) for various amounts of oxygen vacancies. The constant 0.055 in front of the oxygen vacancy content $(2 \delta)$ in the formulae for $t(x, T, \delta)$ depends sensitively on the average equilibrium interatomic distances $\left[\mathrm{Mn}^{3+}-\mathrm{O}\right]$ and $\left[\mathrm{Mn}^{4+}-\mathrm{O}\right]$, and as such may contain

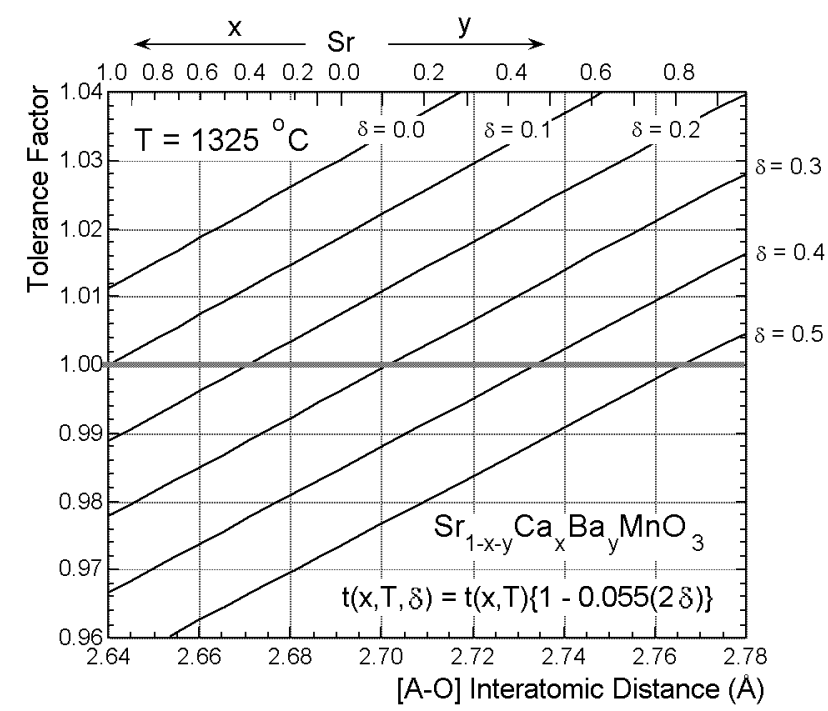

Fig. 2. Calculated tolerance factor $t(x, T, \delta)$ at $T=1325^{\circ} \mathrm{C}$ as a function of the [A-O] bond length (the corresponding composition of $\mathrm{Sr}_{1-x-y} \mathrm{Ca}_{x} \mathrm{Ba}_{y} \mathrm{MnO}_{3}$ is displayed on the upper axis) for various amounts of oxygen vacancies $\delta$. 
a considerable error bar of \pm 0.015 depending on the localized or itinerant state of the $\mathrm{Mn}^{3+}$ and $\mathrm{Mn}^{4+}$ ions. The predicted compositions, at given $T$ and $\delta$ for which the perovskite phase is stable are limited by the condition $t<1$. Despite this condition, it is still possible to obtain kinetically stable perovskite phases with $t>1$ at lower temperatures by a two-step procedure. That procedure produces an oxygen deficient perovskite phase with $t<1$ at elevated temperature followed by cooling in argon or quenching in air without increasing oxygen content and by a low-temperature $\left(T<\sim 600^{\circ} \mathrm{C}\right)$ oxygen anneal that makes $t>1$ without decomposition of the perovskite phase. Knowledge of the functional dependence of the tolerance factor on composition, temperature, and oxygen vacancy concentration guides the preparation of a wide range of new perovskite manganites and other mixed-valent compounds by such a two-step procedure. In the following sections, we will describe several substituted manganite systems for which special synthesis techniques were used to extend chemical composition ranges and selectively order or disorder mixtures of metal atoms on the A- or Mn-sites.

\section{Magnetic transitions and structural features for mixed-valent systems}

Using a modified two-step synthesis method at reduced oxygen pressure followed by low-temperature oxygen anneal, we recently have been able to extend the solubility limits from $x=0.6$ and 0.3 to $x=1$ and 0.7 for the A-site substituted compounds $\mathrm{La}_{1-x} \mathrm{Sr}_{x} \mathrm{MnO}_{3-\delta}$ and $\mathrm{La}_{1-x} \mathrm{Ba}_{x} \mathrm{MnO}_{3-\delta}$, respectively [13, 17]. Recently, we have found that for the large oxygen vacancy content $\delta \sim 0.5$ during the first step of synthesis it is possible to introduce layer ordering of the La and $\mathrm{Ba}$ cations for $0.38 \leq x \leq 0.52$ [18]. Using the two-step synthesis method we have obtained several new $\mathrm{Mn}$-substituted $\mathrm{SrMn}_{1-y} \mathrm{~B}_{y} \mathrm{O}_{3}$ compounds with randomly mixed Mn/B ions. Two such systems will be described here for $\mathrm{B}=\mathrm{Fe}$ $(0 \leq y \leq 1)[19]$ and $\mathrm{Ga}(0 \leq y \leq 0.5)$ [20]. In addition, by controlling the oxygen vacancy content near $\delta \sim 0.5$, it is possible to introduce layer ordering of the $\mathrm{Mn}$ and $\mathrm{Ga}$ ions for $y=0.5$.

Figure 3 shows the complete structure-properties phase diagram for the $\mathrm{La}_{1-x} \mathrm{Sr}_{x} \mathrm{MnO}_{3}$ system [17]. The shadowed area denotes the newly synthesized compositions. A detailed knowledge of the phase diagrams like that of the $\mathrm{La}_{1-x} \mathrm{Sr}_{x} \mathrm{MnO}_{3}$ system is helpful for a comprehensive understanding of the properties of the $\mathrm{Mn}-\mathrm{O}_{3}$ network as a function of the hole doping (band filling) and bond angles (band width). It is also useful for discovering new applicable and improving known properties.

Dramatic changes of the structural and resistive properties in response to the magnetic field were found near the metal-insulator transitions [21]. For example, Fig. 4 shows resistivity of the $x=0.17$ sample in 0 and 3 Tesla. In zero magnetic field, the structural rhombohedral to orthorhombic phase transition at 


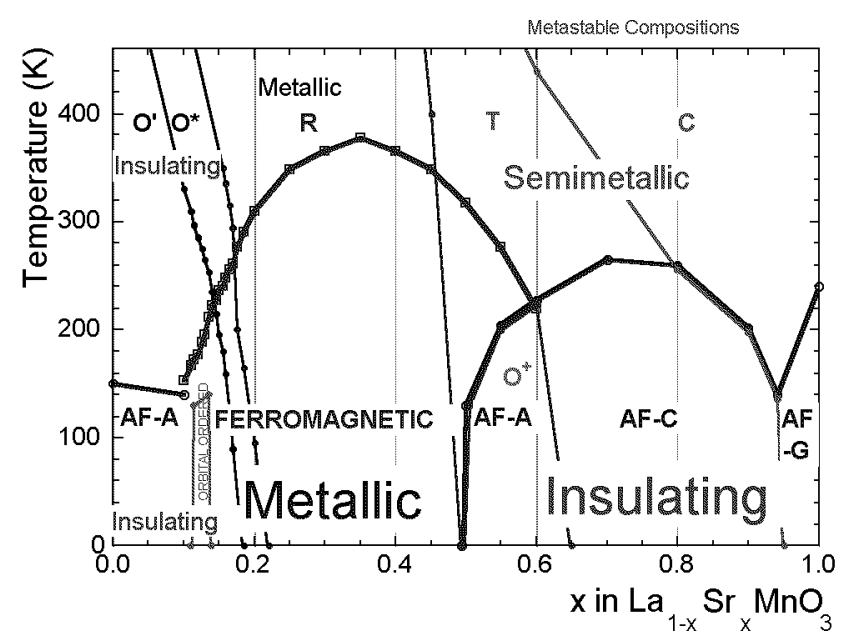

Fig. 3. Composition-properties phase diagram for the $\mathrm{La}_{1-x} \mathrm{Sr}_{x} \mathrm{MnO}_{3}$ system. FM and AF A-, C-, and G-type denote magnetic structures and transition temperatures. Orthorhombic $\left(\mathrm{O}^{\prime}, \mathrm{O}^{*}\right.$, and $\left.\mathrm{O}^{+}\right)$, rhombohedral (R), tetragonal (T), and cubic (C) structural phases and transition temperatures as well as ranges of various conducting properties are also shown.

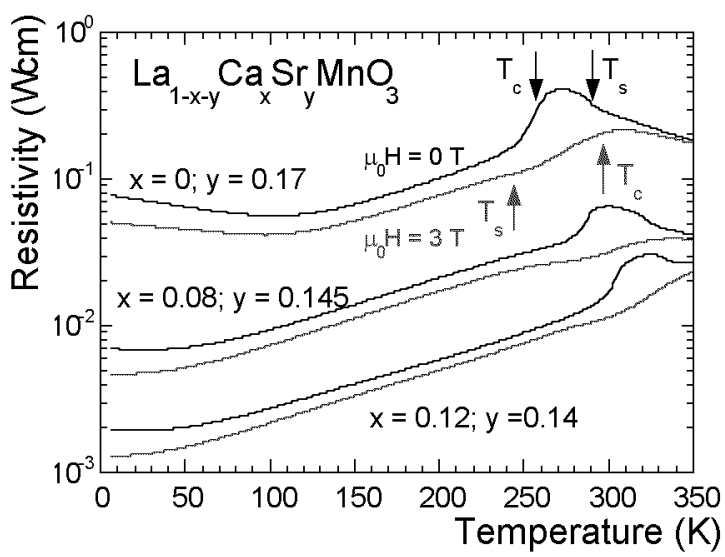

Fig. 4. Resistivity of several $\mathrm{La}_{1-x-y} \mathrm{Ca}_{x} \mathrm{Sr}_{y} \mathrm{MnO}_{3}$ samples near structural (rhombohedral to orthorhombic) transitions in 0 and 3 Tesla. $T_{\mathrm{C}}$ and $T_{5}$ denote ferromagnetic and structural transition temperatures, respectively, in $0 \mathrm{~T}$ (arrow down) and $3 \mathrm{~T}$ (arrow up) for $x=0$ and $y=0.17$.

$T_{\mathrm{s}}=290 \mathrm{~K}$ is slightly above the ferromagnetic transition at $T_{\mathrm{C}}=260 \mathrm{~K}$. Upon cooling, this order of transitions first produces a rapid increase in resistivity at $T_{\mathrm{s}}$ due to enhanced electron scattering by large cooperative JT-distortions, followed by a rapid decrease in resistivity, due to the ferromagnetic transition to metallic phase. By applying a magnetic field, $T_{\mathrm{C}}$ rises rapidly causing the resistive 
increase at $T_{\mathrm{s}}$ to diminish. Above a certain value of the applied field (about $2 \mathrm{~T}$ for $x=0.17), T_{\mathrm{C}}$ is shifted above $T_{\mathrm{s}}$. As a result, a rapid increase in resistivity with decreasing temperature is removed and replaced by a rapid decrease in resistivity at the ferromagnetic transition leading to a significant enhancement of the magnetoresistance. Figure 4 shows an example [22] of several $\mathrm{La}_{1-x-y} \mathrm{Ca}_{x} \mathrm{Sr}_{y} \mathrm{MnO}_{3}$ compositions for which an enhanced CMR effect was achieved by tuning structural and magnetic transitions over a wide temperature interval 260-330 K.

The synthesis of the randomly substituted $\mathrm{La}_{1-x} \mathrm{Ba}_{x} \mathrm{MnO}_{3}$ system required a more complicated procedure. This was done at high temperatures $\left(1300-1400^{\circ} \mathrm{C}\right)$ using Ar flow to achieve the oxygen-deficient compositions $\mathrm{O}_{2.6-2.8}$ followed by the complete oxygenation (air or $\mathrm{O}_{2}$ flow) at a temperature low enough (below $500^{\circ} \mathrm{C}$ ) that the cations do not diffuse. The formation of a well-layered-ordered $\mathrm{LaBaMn}_{2} \mathrm{O}_{6-\delta}$ phase at high temperatures required accurate control of the oxygen content at $\mathrm{O}_{5}$ using in situ TGA apparatus in $\mathrm{H}_{2} / \mathrm{Ar}$ flow [18]. A comparison of layered-ordered and randomly substituted compounds with the same chemical compositions (i.e., the same charge doping and tolerance factor) revealed much greater effects of the charge disorder on the ferromagnetic transition temperature for mixed valent perovskite manganites than was apparent for the single-valent $\mathrm{Sr}_{1-x} \mathrm{Ca}_{x} \mathrm{MnO}_{3}$ system [13, 15]. Because our measured interatomic distances of $[\mathrm{La}-\mathrm{O}]=2.765$ and $[\mathrm{Ba}-\mathrm{O}]=2.783 \AA$ are quite similar, the variance of size is small $s(x=0.5)=0.009 \AA$, i.e., the effects of structural disorder are anticipated to be small. Nonetheless, we observe a large, $\sim 80 \mathrm{~K}$, difference of ferromagnetic $T_{\mathrm{C}}$ between the two structural forms. We attribute the different properties of the randomly substituted and layered-ordered phases to the local A-site charge disordering that profoundly disturbs the coherent electronic states of the $\mathrm{Mn}-\mathrm{O}$ bonds. The difference between $T_{\mathrm{C}}$ 's of the two structural forms decreases for compositions $0.38 \leq x<0.50$ because of the introduction of structural and charge disorder for the layer-ordered phase with a partial substitution of $\mathrm{La}$ in the layers of $\mathrm{Ba}-\mathrm{O}$. Nonetheless, the newly synthesized layer-ordered phase with composition $x=0.42$ shows the second highest FM transition temperature of $365 \mathrm{~K}$ ever observed for the perovskite manganites. Similar effects of local structural/charge order no doubt have profound effects on the properties of other complex oxides; for example, layered copper oxide high temperature superconductors.

Figure 5 shows magnetic phase diagram for the $\mathrm{SrMn}_{1-y} \mathrm{Fe}_{y} \mathrm{O}_{3}$ compounds constructed by combination of structural, magnetic, and transport measurements with Mössbauer spectroscopy [19]. The complete solid solution was achieved for this system with unusual simple cubic structure, i.e., with straight average bonds angles. We have observed only random mixing of $\mathrm{Mn}$ and $\mathrm{Fe}$ and could not induce any kind of $\mathrm{Mn} / \mathrm{Fe}$ ordering for $\mathrm{SrMn}_{0.5} \mathrm{Fe}_{0.5} \mathrm{O}_{3-\delta}$ over the entire range of $\delta=0-0.5$. We have found the AF ordering for lightly and heavily Fe-substituted material, while intermediate substitution leads to spin-glass behavior. Near the composition $\mathrm{SrMn}_{0.5} \mathrm{Fe}_{0.5} \mathrm{O}_{3}$, these two types of ordering are found to coexist and 


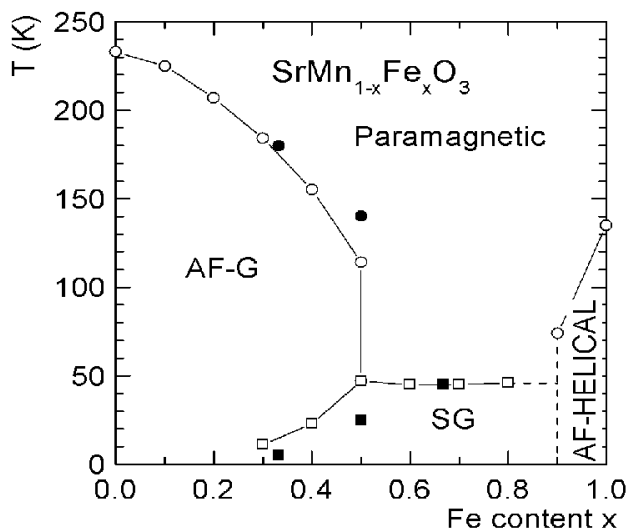

Fig. 5. Composition-magnetic properties phase diagram for the $\mathrm{SrMn}_{1-y} \mathrm{Fe}_{y} \mathrm{O}_{3}$ compounds. Solid markers are data points for oxygen deficient samples from Ref. [23].

affect one another. The spin-glass behavior may be caused by the competing FM and $\mathrm{AF}$ interactions among randomly substituted $\mathrm{Mn}^{4+}$ and observed $\mathrm{Fe}^{3+}$ and $\mathrm{Fe}^{5+}$ ions. The same competition and disorder may cause gradual suppression of $T_{\mathrm{N}}$ with Fe substitution.

A similar suppression of $T_{\mathrm{N}}$ and the spin-glass behavior at $y=0.5$ was observed for the randomly substituted $\mathrm{SrMn}_{1-y} \mathrm{Ga}_{y} \mathrm{O}_{3-y / 2}$ compounds. These compounds in the composition range $0 \leq y \leq 0.3$ can be prepared by heating in $\mathrm{Ar}$ at $1300^{\circ} \mathrm{C}$ followed by oxygen annealing that brings $\mathrm{Mn}$ oxidation state to $4+$, i.e., the maximum oxygen content is $3-y / 2$. However, to make $0.3<y \leq 0.5$ requires the TGA synthesis in $1 \% \mathrm{O}_{2} / \mathrm{Ar}$ such that the oxygen content is $\sim 2.55$ at the synthesis temperature. At higher oxygen content, the perovskite phase is not stable, while at lower oxygen content, $y=0.4$ sample is multi-phase, and for $y=0.5$ the single-phase layer-ordered $(\mathrm{Mn} / \mathrm{Ga})$ is formed. Thus, similar to the $\mathrm{LaBaMn}_{2} \mathrm{O}_{6-\delta}$ phases, it is possible to order or disorder cations and oxygen vacancies in the perovskite structure. These ordering phenomena have profound effects on properties; for example, the randomly substituted $\mathrm{SrMn}_{0.5} \mathrm{Ga}_{0.5} \mathrm{O}_{2.75}$ shows spin-glass behavior at $20 \mathrm{~K}$, the layer-ordered $\mathrm{Sr}_{2} \mathrm{MnGaO}_{5}$ is G-type $\mathrm{AF}$ at $80 \mathrm{~K}$, and the layer-ordered $\mathrm{Sr}_{2} \mathrm{MnGaO}_{5.5}$ is mixed C- and G-type AF at 130 and $90 \mathrm{~K}$, respectively.

\section{Conclusions}

Bond angles $\mathrm{Mn}-\mathrm{O}-\mathrm{Mn}$ and bond lengths $[\mathrm{A}-\mathrm{O}]$ have been used to describe $T_{\mathrm{N}}$ of the single-valent manganites. The maximum $T_{\mathrm{N}}$ was found for straight bonds $\left(\cos ^{2}(\theta) \sim 1\right)$ and minimal variance of sizes $(s \sim 0)$. The tolerance factor $t(x, T, \delta)$ have been used to describe the stability of the perovskite phase at the synthesis temperature, identify structural properties at room temperature, and develop the 
design rules for controlling properties of novel compounds. We have shown that the thermogravimetric synthesis can be used to greatly extend solubility limits and obtain new A- and Mn-site ordered perovskites. The study of the correlations between chemical, structural, and physical properties for mixed-valent manganites show that $T_{\mathrm{C}}$ and $T_{\mathrm{N}}$ depend strongly on the tolerance factor $(t)$, charge doping $(h)$, structural disorder $(s)$, and charge disorder.

\section{Acknowledgment}

The work at NIU was supported by the NSF-DMR-0302617 and by the State of Illinois under HECA. At ANL the work was supported by the U.S. Department of Energy, Division of Basic Energy Science - Materials Sciences, under contract No. W-31-109-ENG-38.

\section{References}

[1] Y. Tokura, N. Nagaosa, Science 288 (5465), 462 (2000).

[2] G.F. Dionne, in: Magnetic Interactions and Spin Transport, Eds. A. Chtchelkanova, S. Wolf, Y. Idzerda, Kluwer Academic, 2003, p. 1.

[3] V.M. Goldshmidt, Skrifter Norske Videnskaps-Akad. Oslo, I. Mat.-Naturv. Kl. No. 8 (1926).

[4] R.D. Shannon, Acta Crystallogr. A 32, 751 (1976).

[5] B. Dabrowski, O. Chmaissem, J. Mais, S. Kolesnik, J.D. Jorgensen, S. Short, J. Solid State Chem. 170, 154 (2003).

[6] C.J. Ballhausen, Introduction to Ligand Field Theory, McGraw-Hill, New York 1962.

[7] J.B. Goodenough, Magnetism and Chemical Bond, Interscience Publishers, John Wiley, New York 1963.

[8] C. Boekema, F. Van Der Woude, G.A. Sawatzky, Int. J. Magnetism 3, 341 (1972).

[9] J.B. Goodenough, J.M. Longo, in: Landolt-Bornstein Tabellen, New Series III/4a, Ed. K. Hellwege, Springer, Berlin 1970, p. 231.

[10] O. Chmaissem, B. Dabrowski, S. Kolesnik, J. Mais, D.E. Brown, R. Kruk, P. Prior, B. Pyles, J.D. Jorgensen, Phys. Rev. B 64, 134412 (2001).

[11] J.B. Goodenough, Phys. Rev. 100, 564 (1955).

[12] M.L. Medarde, J. Phys., Condens. Matter 9, 1679 (1997).

[13] B. Dabrowski, O. Chmaissem, M. Mais, S. Kolesnik, J.D. Jorgensen, S. Short, Mat. Res. Soc. Symp. Proc. 718, 169 (2002).

[14] L.M. Rodriguez-Martinez, J.P. Attfield, Phys. Rev. B 54, R15622 (1996).

[15] B. Dabrowski, O. Chmaissem, S. Kolesnik, M. Mais, J.D. Jorgensen, Physica C 387, 266 (2003).

[16] J.B. Torrance, P. Lacorre, A.I. Nazzal, E. J. Ansaldo, Ch. Niedermayer, Phys. Rev. $B$ 45, 8209 (1992). 
[17] O. Chmaissem, B. Dabrowski, S. Kolesnik, M. Mais, J.D. Jorgensen, S. Short, Phys. Rev. B 67, 094431 (2003).

[18] B. Dabrowski, O. Chmaissem, J. Mais, S. Kolesnik, J.D. Jorgensen, unpublished.

[19] S. Kolesnik, B. Dabrowski, J. Mais, D.E. Brown, O. Chmaissem, R. Kruk, C.W. Kimball, Phys. Rev. B 67, 144402 (2003).

[20] A. Caspi, M. Adeev, J.D. Jorgensen, S. Short, B. Dabrowski, O. Chmaissem, J. Mais, S. Kolesnik, unpublished.

[21] B. Dabrowski, X. Xiong, Z. Bukowski, R. Dybzinski, P.W. Klamut, J.E. Siewenie, C.W. Kimball, O. Chmaissem, J.D. Jorgensen, Phys. Rev. B 60, 7006 (1999).

[22] S. Kolesnik, B. Dabrowski, Z. Bukowski, J. Mais, J. Appl. Phys. 89, 1271 (2001).

[23] I.D. Fawcett, G.M. Veith, M. Greenblatt, M. Croft, I. Novik, Solid State Sci. 2, $821(2000)$. 